IP Periodica Polytechnica Civil Engineering

62(4), pp. 866-872, 2018

https://doi.org/10.3311/PPci.9424

Creative Commons Attribution (i)

RESEARCH ARTICLE

\section{Effect of Sodium Carbonate Concentration in Calgon on Hydrometer Analysis Results}

\author{
Arshdeep Kaur $^{1 *}$, George C. Fanourakis ${ }^{1}$
}

Received 03 May 2016; Revised 29 July 2017; Accepted 22 March 2018

\begin{abstract}
Calgon (a combination of sodium hexametaphosphate and sodium carbonate) has proved to be the most effective dispersing agent in determining the grain size distribution of finegrained soils by means of the hydrometer analysis. Previous research on the effect of the sodium hexametaphosphate content of dispersing agents on the clay contents showed that the addition of sodium carbonate to sodium hexametaphosphate increases its dispersing effectiveness. Hence, Calgon 35:7 was used /recommended by many researchers/methods and proved to be the most effective dispersing agent. Although previous work focusing on the effect of varying the concentration of sodium hexametaphosphate in Calgon has been reported, the effect of the concentration of sodium carbonate in Calgon has not been assessed and reported. For this reason, in this investigation a series of hydrometer test analyses were conducted using the 152H and ASTM 152H: E100 hydrometers with Calgon in ratios of 35:0, 35:20 and 35:30. It was observed that with any increase in sodium carbonate content beyond 7 grams, the percentage clay content actually decreased tremendously in the case of hydrometer $152 \mathrm{H}$. However, for the other hydrometer, Calgon (35:0) proved to be most effective combination. Thus, the increase in the sodium carbonate content in Calgon, beyond $7 \mathrm{~g} /$ litre, is not recommended.
\end{abstract}

\section{Keywords}

grain-size analysis, calgon, sodium carbonate, hydrometer tests, TMH1 (1986)

\section{Introduction}

Soils consist of various particle sizes and shapes. The particle size analysis is a method of separating the soil into different fractions, based on the sizes of particles present in the soil. The particle size analysis is divided into two categories (coarse and fine) with associated laboratory test methods. A sieve analysis is used to separate the coarse-grained fraction of soil i.e., the fraction of the soil with particle size greater than 425 micron. Whereas, the sedimentation analysis is used for the analysis of the fine-grained fraction of the soil, such as silt and clay, of which particle size is less than 75 microns. Various researchers have investigated the effectiveness of the determination of the particle size analysis, on the fine fraction of various soils, using different methods such as the hydrometer (sedimentation) method ([1], [2], [3], [4], [5], [6], [7], [8], [9], [10], [11], [12], $[13])$, the pipette method ([8], [14], [15]) and the laser diffraction method ([6], [9], [11], [15], [16], [17], [18], [19], [20]).

This study however focused on the hydrometer analysis, as in South Africa, the national laboratories use the hydrometer analysis to determine the particle size distribution of the fines fraction of soils. In addition, the hydrometer method [13] is still in widespread use, as it requires inexpensive equipment and only basic technical expertise. However, in South Africa, there is a problem with the accurate determination of the clay content of soils, which was reflected in the results obtained from different laboratories that analyzed the samples. This problem, which was formally expressed by Jacobsz and Day (2008) [21], justifies the need for research into all the variables of the hydrometer test, with a view to improving its accuracy and perhaps standardize the test nationally and possibly, in future, internationally. The accurate determination of the clay content of a soil is important as it is used to determine its activity, which in turn is used for design purposes. Inaccurate clay content determinations have resulted in inappropriate design solutions, which have even led to unacceptable damage to the structures. The TMH1 method [22] is usually applied in South Africa for the determination of the particle size distribution of soils. However, this standard has been superseded by a different method, SANS 3001 [23], which has not been adopted by most laboratories. Almost 
all the national laboratories follow the old method [22], with a deviation in the type of dispersing agent. Dispersing agents are used to disperse the fine-grained particles of the soil in the suspension medium (water) in the hydrometer analysis. The old method [22] prescribes the use of a combination of sodium silicate and sodium oxalate as the dispersing agent. However, this dispersing agent is often substituted with other dispersing agents such as Calgon or sodium pyrophosphate decahydrate (still used by Soil Laboratory, South Africa) or sodium tetra pyrophosphate (formerly used by the laboratory of Multitoets, South Africa) or Di-sodium Di-hydrogen pyrophosphate (formerly used by the Soils Testing Laboratory of Department of Water Affairs of South Africa) or sodium hexametaphosphate (still used by Soillab, South Africa).

In an attempt to study the geotechnical properties (mainly the clay content) of the soils naturally found in South Africa, an exhaustive study was undertaken [1] to determine the most effective dispersing agent in terms of type, optimum concentration and volume used on the percentage clay yielded. A volume of $80 \mathrm{ml}$ of Calgon 35:7 proved to be the most effective dispersing agent (when testing was carried out with different volumes and concentration of Calgon by varying only the concentration of sodium hexametaphosphate in the Calgon) in the case of one of the samples (black soil), yielding a clay content of $35.2 \%$ [1]. In the case of another sample (red soil), $20 \mathrm{ml}$ of Sodium tetra pyrophosphate yielded the maximum clay content of $32.3 \%$, whereas $29 \%$ clay content was yielded by 125 $\mathrm{ml}$ of Calgon 35:7 [2]. All these results were computed by considering the effect of the dispersing agent on the hydrometer reading, and appropriately correcting the hydrometer readings by subtracting the readings obtained on companion "blank" solutions containing only water and the relevant dispersing agent (no soil), at relevant time period, from the reading taken on the solution containing water, soil and dispersing agent.

TMH1 (1986) [22] does not make any provision for the effect of dispersing agent in the solution on the readings recorded during the test and it was observed that with the change in the type of dispersing agent, the method was actually incorrectly yielding inflated fines (including clay) contents. Hence, ideally all the national laboratories, which follow the TMH1 (1986) method [22] with a deviation in the dispersing agent type, should consider correcting the hydrometer test readings, if this is not being done.

A study on the effect of different types and quantities of dispersing agents on the grain size distribution, particularly the percentage of clay size, was described by Sridharan et al [4]. They concluded that clay size fraction could vary from $4 \%-45 \%$ for marine clays, depending upon the dispersing agent used. It was further seen that $100-125 \mathrm{ml}$ of Calgon 33:7 (33 grams of Sodium hexametaphosphate and 7 grams of Sodium carbonate in 1 litre of distilled water) was found to be the most effective dispersing agent.
Bindu and Ramabhadran [5] conducted a study to evaluate the effect of the concentration of the dispersing agent on the hydrometer analysis and attempted to optimize the concentration of the dispersing agent to be added to obtain maximum dispersion. It was observed that the addition of sodium carbonate improved the dispersing capacity of sodium hexametaphosphate. The optimum volume and concentration was found to be $100 \mathrm{ml}$ of $6 \%(53: 7)$ and there was a significant decrease in dispersion with a further increase in the concentration as well as the volume of the dispersing agent added.

Emeka [10] conducted a hydrometer analysis study on two soil samples naturally available in Nigeria using four solutions (100 $\mathrm{ml}$ of Calgon (35:7), $125 \mathrm{ml}$ of $4 \%$ of Sodium hexametaphosphate, $14 \%$ of sodium carbonate and no dispersing agent). It was observed that both calgon and sodium hexametaphosphate proved to be consistent solutions whereas the sodium carbonate solutions did not effectively disperse both the samples.

Calgon, which is a combination of sodium hexametaphosphate and sodium carbonate, is one of the popular dispersing agents adopted by various countries in the sedimentation test analysis. This dispersing agent in different concentrations (varying the amount of only sodium hexametaphosphate in Calgon) is recommended and adopted by many methods and researchers ([1], [2], [3], [4], [5], [10], [24], [25], [26]).

However, the effect of variation of the amount of sodium carbonate in Calgon on the magnitude of clay content determined by the hydrometer analysis is still to be explored. Bindu and Ramabhadran [5] concluded that the addition of sodium carbonate improved the dispersion capacity of sodium hexametaphosphate, but they carried out the testing by adding only 7 grams of sodium carbonate with different masses of sodium hexametaphosphate (13 grams, 33 grams, 53 grams and 73 grams). In the past, no work has been reported pertaining to various concentrations of sodium carbonate in the Calgon. Hence, the primary purpose of this investigation was to assess the effect of the amount of sodium carbonate in Calgon on the clay content yielded by a series of hydrometer tests, performed on two South African soils. The effect of the hydrometer type was also assessed by incorporating two types of hydrometers (152H and ASTM 152H: E100) into the test program.

\section{Laboratory tests, materials and methodology 2.1 Materials}

Soil Samples: Two soil samples were collected from various parts of South Africa, which comprised of a black soil from the town of Brits in the North-west Province and a red soil sample from Springfield (near Springs) in the Gauteng area. The black soil is a residual soil derived from Norite rock and the typical predominant clay type in the soil is Montmorillonite, whereas the geological origin of the red soil was not established. The Atterberg Limits and Activity of these soils were determined, at the laboratories of the Department of Civil Engineering Technology, 
as shown in Table 1. The TMH1 (1986) method [22] was used for the determination of the Liquid Limit and Plastic Limit. The clay content in Table 1 was determined by the means of the hydrometer analysis by following TMH1 (1986) guidelines [22] with a deviation in the prescribed dispersing agent. TMH1 (1986) Method A6 [22] prescribes $5 \mathrm{ml}$ of sodium silicate and $5 \mathrm{ml}$ of sodium oxalate as the dispersing agent. However, 125 $\mathrm{ml}$ of Calgon 35:7 (a solution comprising 35 grams of sodium hexametaphosphate (NaHMP) and 7 grams of sodium carbonate $\left(\mathrm{Na}_{2} \mathrm{CO}_{3}\right)$ in 1 litre of distilled water) was used. Furthermore, the hydrometer readings were appropriately adjusted by subtracting the hydrometer readings obtained on the "blank" companion specimens to account for the effect of the dispersing agent.

Table 1 Atterberg limits and Activity of the soils sampled

\begin{tabular}{lcc}
\hline Properties & Black Soil & Red Soil \\
\hline Liquid Limit (LL) & 56 & 28.3 \\
Plastic Limit (PL) & 22 & 15.1 \\
Plasticity Index (PI) & 34 & 13.2 \\
Clay Content (\%) & 32 & 29 \\
Activity (A) & 1.07 & 0.5 \\
\hline
\end{tabular}

Dispersing Agents: The hydrometer tests were performed by varying the amount of sodium carbonate to 0 grams, 20 grams and 30 grams from usual 7 grams in Calgon (keeping the amount of sodium hexametaphosphate constant as 35 grams) on the two soil samples, following the THM1 (1986) test method [22].

Hence, the amounts of 3.5\% (Calgon 35:0), 5.5\% (Calgon $35: 20$ ) and $6.5 \%$ (Calgon 35:30) solution of Calgon were prepared, by mixing the required quantity in 1 litre of distilled water. The results obtained from these concentrations were compared with the results obtained by Calgon (35:7), which was used as the reference dispersing agent. The quantities (in grams) of chemicals added for the preparation of these stock solutions are given in Table 2.

Table 2 Quantity of chemicals added for preparation of Calgon solutions

\begin{tabular}{lcc}
\hline $\begin{array}{l}\text { Concentration of } \\
\text { Solution }(\%)\end{array}$ & $\begin{array}{c}\text { Calgon } \\
\text { Quantity of NaHMP } \\
\text { Added }(\mathrm{g})\end{array}$ & $\begin{array}{c}\text { Quantity of } \mathrm{Na}_{2} \mathrm{CO}_{3} \\
\text { Added }(\mathrm{g})\end{array}$ \\
\hline 3.5 & 35 & 0 \\
5.5 & 35 & 20 \\
6.5 & 35 & 30 \\
\hline
\end{tabular}

In most of these tests the minimum volume of Calgon initially used was $100 \mathrm{ml}$. The justification for this quantity is that methods [24] and [25] recommend $100 \mathrm{ml}$ and $125 \mathrm{ml}$ of Calgon solution, respectively and hence $100 \mathrm{ml}$ volume was selected to confirm the recommended volume. However, this volume was incrementally increased or decreased until the optimum volume for each concentration was established. In a few cases the minimum volume of Calgon was even reduced to $25 \mathrm{ml}$ to get the desirable results.
Hydrometers: TMH1 (1986) [22] prescribes the use of hydrometer $152 \mathrm{H}$, but the authors [3] concluded that the 152H:E100 was more effective by yielding higher clay contents in the case of the more active soil. Therefore, both hydrometers were incorporated into this investigation i.e. $152 \mathrm{H}$ and ASTM 152H:E100.

\subsection{Testing procedure and calculations}

For the current testing, the guidelines laid down by TMH1 (1986) [22] were followed. Figure 1 shows the details of the hydrometer tests including the determination of the percentage finer than the various sizes. The procedure detailed in this figure was followed using the Bouyoucos $(152 \mathrm{H})$ hydrometer as well as the ASTM 152H: E100 (H2) hydrometer. The procedure is discussed below. For all the tests performed, 50 grams of soil sample passing through 425 micron sieve was mixed with the desired quantity of dispersing agent and about $400 \mathrm{ml}$ of distilled water in a canning jar. The soil water mixture was allowed to stand overnight. After the mixture had been allowed to stand, it was dispersed for 15 minutes with a standard paddle. The paddle was washed clean with distilled water allowing the wash water to run into a container with the suspension.

The suspension was then poured into the Bouyoucos cylinder and the canning jar was rinsed with distilled water from the wash bottle. The cylinder was then filled with distilled water to $1130 \mathrm{ml}$ mark with the Bouyoucos hydrometer $(152 \mathrm{H})$ inside. Thereafter, the hydrometer was removed and the cylinder was inverted a few times, using the palm of one hand as a stopper over the mouth of the cylinder to ensure that the temperature was uniform throughout. After bringing the cylinder to vertical position, a stopwatch was started. The hydrometer was inserted and readings were taken at 18 and $40 \mathrm{sec}-$ onds without removing the hydrometer from the cylinder. The hydrometer was then taken out and rinsed with water and it was again inserted into suspension when the elapsed time was 2 minutes. This reading was noted and the hydrometer was removed and placed in the distilled water. This procedure was repeated for the 5 minutes, 15 minutes, 30 minutes, 1 hour, 4 hour and 24 hours readings. After taking each hydrometer reading, position, a stopwatch was started. The hydrometer was inserted and readings were taken at 18 and 40 seconds without the temperature of the liquid was recorded.

Temperature corrections were applied to the readings. The same procedure was followed for the tests performed with the other hydrometer (ASTM 152H: E100) as well. This hydrometer is referred to as $\mathrm{H} 2$ in the discussion of the results.

In addition, a blank solution comprising distilled water and dispersing agent (no soil) was prepared, in a second bouyoucos cylinder in the same proportions as the solutions prepared with the soil. The dispersing agent and water mixture was also soaked overnight and identical hydrometer tests were performed for the blank solutions. 


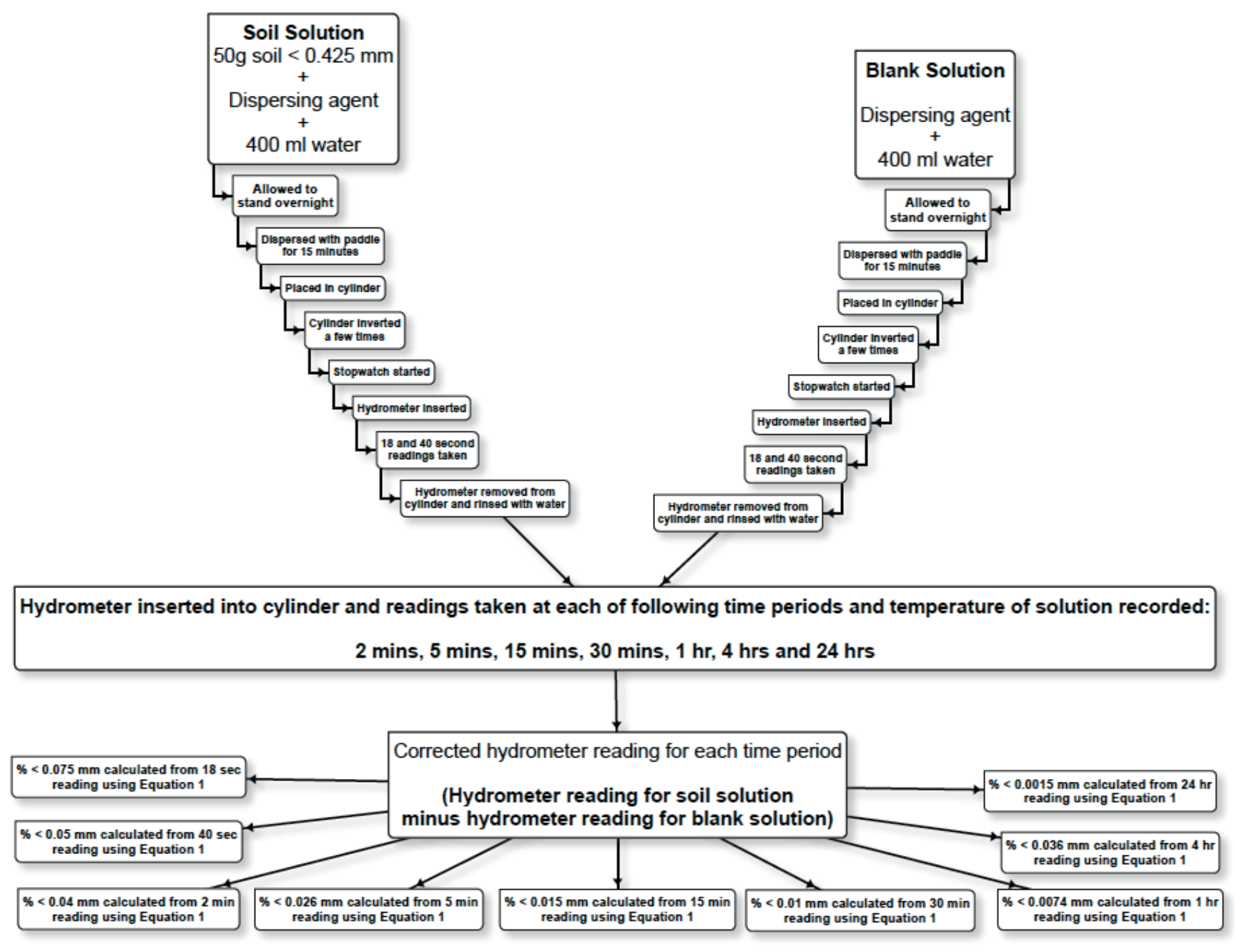

Fig. 1 Details of hydrometer tests

The hydrometer readings taken on the samples which contained soil were appropriately adjusted by subtracting the hydrometer readings obtained on the "blank" companion specimens, at the relevant times. This accounted for the effect of the dispersing agent on the readings. It should be noted that the TMH1 (1986) method [22] does not make any provisions for this correction.

The percentages finer than $0.075 \mathrm{~mm}, 0.05 \mathrm{~mm}, 0.04 \mathrm{~mm}$, $0.026 \mathrm{~mm}, 0.015 \mathrm{~mm}, 0.01 \mathrm{~mm}, 0.0074 \mathrm{~mm}, 0.0036 \mathrm{~mm}$ and $0.0015 \mathrm{~mm}$ were respectively calculated by the readings taken at $18 \mathrm{sec}, 40 \mathrm{sec}, 2 \mathrm{~min}, 5 \mathrm{~min}, 15 \mathrm{~min}, 30 \mathrm{~min}, 1$ hour, 4 hour and 24 hours, by means of Equation 1.

$$
P=\left(C \times S_{f}\right) / S_{h}
$$

Where, $P=$ Percentage finer than relevant size,

$S_{h}=$ Mass of soil fines used in analysis (50 grams),

$S_{f}=$ Percentage soil fines in total sample $(<0.425 \mathrm{~mm})$,

$C=$ Corrected hydrometer reading

The percentage clay content present in each sample (fraction finer than $0.002 \mathrm{~mm}$ ) was obtained from the relevant particle size distribution curve.

\section{Results and discussion}

\subsection{Black soil}

Figure 2 represents the effect of different concentrations of sodium carbonate in Calgon and volumes of Calgon on the clay content of the black soil, determined by hydrometer analysis, using the two hydrometer types.

Figure 2 clearly show that the following.

- The $4.2 \%$ Calgon (35:7) was the most effective dispersing agent yielding a maximum clay content of $35 \%$ for the black soil when the testing is done with hydrometer $152 \mathrm{H}$. Thus increasing the amount of sodium carbonate was not effective in this case. Emeka [10] also concluded that Calgon (35:7) is a consistent solution.

- When the tests were performed with hydrometer $152 \mathrm{H}$ : E100 (H2), 3.5\% Calgon (35:0) yielded the maximum clay content of $26.7 \%$. This is in disagreement with Bindu and Ramabhadran [5], as they indicated that the addition of sodium carbonate increases the dispersing capacity of sodium hexametaphosphate.

- For both the hydrometers, for all the concentrations of dispersing agent, any further increase in the volume of chemical after attaining optimum volume generally resulted in a 


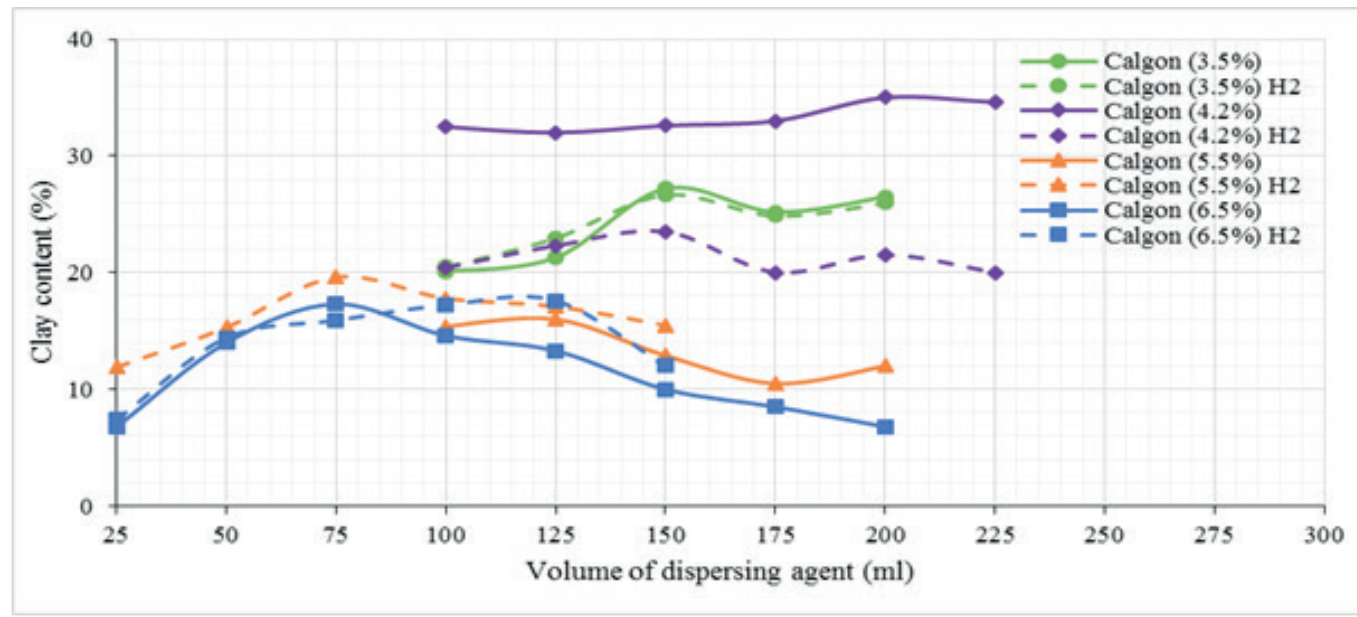

Fig. 2 Effect of sodium carbonate concentration in Calgon and volume of Calgon on the clay content of Black Soil.

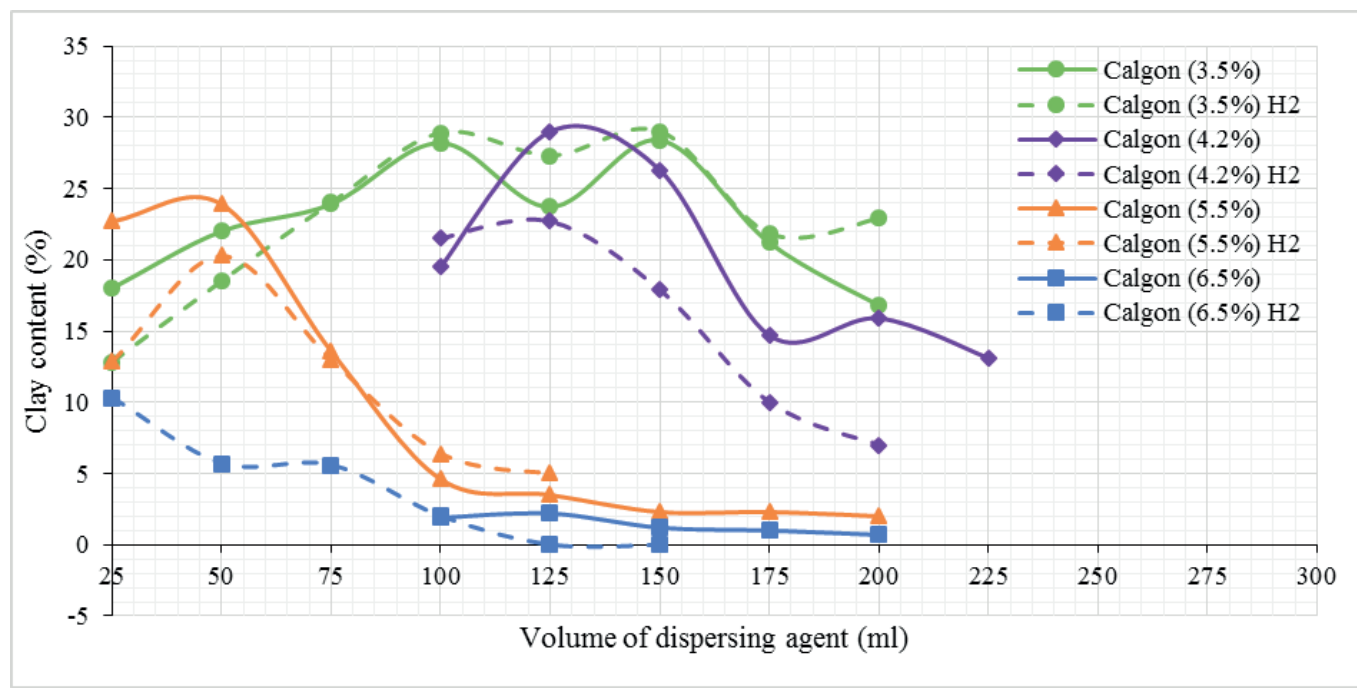

Fig. 3 Effect of sodium carbonate concentration in Calgon and volume of Calgon on the clay content of Red Soil.

decrease in clay content. Similar results were obtained by other researchers as well ([1], [2], [3], [4], [5]).

- Relatively high sodium carbonate contents $(5.5 \%$ and 6.5 $\%$ ) resulted in a decrease in the clay contents and rendered the hydrometer $152 \mathrm{H}$ : E100 $(\mathrm{H} 2)$ to be more effective. The reason behind this finding is still not known.

\subsection{Red soil}

Figure 3 represents the effect of different concentrations of sodium carbonate in Calgon and volumes of Calgon on the clay content of the red soil, determined by hydrometer analysis, using the two hydrometer types.

The following considerations can be described according to the Fig. 3.

- The $4.2 \%$ Calgon (35:7) is still the best dispersing agent yielding the maximum clay content of $29 \%$ for the red soil when the testing is done with hydrometer $152 \mathrm{H}$. This was the case in the Black soil as well that, increasing the amount of sodium carbonate did not increase the effectiveness of the dispersing agent. Emeka [10] also concluded that Calgon (35:7) is a consistent solution.
- When the tests were performed with the hydrometer $152 \mathrm{H}$ : E100, $150 \mathrm{ml}$ of 3.5\% Calgon (35:0) yielded the maximum clay content of $29 \%$ however $100 \mathrm{ml}$ of $3.5 \%$ Calgon (35:0) yielded $28.9 \%$ of clay content. Hence, $100 \mathrm{ml}$ of $3.5 \%$ Calgon may be considered as the optimum volume. These results are in disagreement with Bindu and Ramabhadran [5] as they indicated that the addition of sodium carbonate increased the dispersing capacity of sodium hexametaphosphate.

- There is decrease in the clay contents of the red soil after the optimum concentration and volume for each hydrometer is reached and this is actually high in case of hydrometer $152 \mathrm{H}$.

- Also 6.5\% Calgon was relatively less effective in the case of both the hydrometers, yielding even zero readings in case of hydrometer 152H: E100. The percentage clay content obtained at optimum volume decreased tremendously (90.8\% for hydrometer $152 \mathrm{H}$ and $49.3 \%$ for ASTM $152 \mathrm{H}$ : E100 hydrometer) when the concentration of Calgon was increased from $5.5 \%$ to $6.5 \%$.

- For both the hydrometers, for all the concentrations of dispersing agent, any further increase in the volume of chemical after attaining optimum volume generally resulted in 
a decrease in clay content. Similar results were obtained by other researchers [1], [2], [3], [4], [5]. However, a contradictory trend was noticed where, with an increase in volume there was an increase in percentage of clay content (3.5\% Calgon (35:0)). The reason for this is that with an increase in the volume of dispersing agent in the companion "blank" solutions, the hydrometer readings increased but the increase was not constant.

\section{Conclusions}

The following observations and conclusions were made regarding the effect of the quantity of sodium carbonate in Calgon, in the hydrometer analysis, on the clay contents yielded for both the black and red soil samples:

1. Tests on soil samples with Calgon as dispersing agent clearly indicated that the percentage of clay size material decreased significantly when amount of sodium carbonate in Calgon increased beyond 7 grams for both types of hydrometer.

2 . In case of both the soils, for the hydrometer $152 \mathrm{H}$, the optimum concentration of Calgon was found to be $4.2 \%$ of the solution. Further increases in sodium carbonate yielded lower clay contents. The percentage decrease was found to be $54 \%$ and $17.6 \%$ for black and red soil, respectively.

3. When the ASTM hydrometer 152H: E100 (H2) was utilized for recording the readings, any addition of sodium carbonate to Calgon solution actually reduced the effectiveness, thus yielding lower results in comparison to the results obtained with 3.5\% Sodium hexametaphosphate or $3.5 \%$ Calgon (35:0). The percentage decrease in clay content beyond the optimum concentration of $3.5 \%$ was $12 \%$ and $21.7 \%$ for black and red soil, respectively.

4. $6.5 \%$ Calgon was the least effective concentration of dispersing agent.

5. No trend was established regarding the accuracy of each hydrometer type when used with a particular dispersing agent. However, the hydrometer $152 \mathrm{H}$ (H1) was generally more effective in the case of the tests conducted on the red soil.

6. When considering each dispersing agent, the optimum volumes were lower in the case of the red soil, which had a lower activity (0.5) than the black soil (1.07).

Finally, the effect of variation of amount of sodium carbonate beyond the recommended 7 grams proved to be ineffective, particularly in the case of higher concentrations and hence is not recommended

\section{References}

[1] Kaur, A., Fanourakis, G. C. "The effect of type, concentration and volume of dispersing agent on the magnitude of the clay content determined by the hydrometer analysis". Journal of South African Institution of Civil Engineers, 58(4), pp. 48-54. 2016.

https://doi.org/10.17159/2309-8775/2016/v58n4a5
[2] Kaur, A., Fanourakis, G. C. "A further investigation of the effect of type, concentration and volume of dispersing agent on the magnitude of the clay content determined by the hydrometer analysis". Journal of South African Institution of Civil Engineers, (Under review). 2016 (b).

[3] Kaur, A., Fanourakis, G. C. "Effect of hydrometer type on particle size distribution of fine grained soil". In: Proceedings of First Southern African Geotechnical Conference. Sun City, South Africa. pp. 307-315. 2016. https://doi.org/10.1201/b21335-55

[4] Sridharan, A., Jose, B. T., Abraham, B. M. "Determination of clay size fraction of marine clays". Geotechnical Testing Journal, 14(1), pp. 103-107. 1991.

https://doi.org/10.1520/GTJ10197J

[5] Bindu, J., Ramabhadran, A. "Effect of concentration of dispersing agent on the grain size distribution of fine grained soil". In: Proceedings of Indian Geotechnical Conference. Mumbai, India. pp. 275-278. 2010. https:// gndec.ac.in/ igs/ldh/conf/2010/articles/067.pdf

[6] Lu, N., Ristow, G. H., Likos, W. J. "The accuracy of hydrometer analysis for fine-grained clay particles". Geotechnical Testing Journal, 23(4), pp. 487-495. 2000.

https://doi.org/10.1520/GTJ11069J

[7] Ashworth, J., Keyes, D., Kirk, R., Lessard, R. "Standard procedure in the hydrometer method for particle size analysis". Communications in Soil Science and Plant Analysis, 32(5-6), pp. 633-642. 2001. https://doi.org/10.1081/CSS-100103897

[8] Kettler, T. A., Doran, J. W., Gilbert, T. L. "Simplified Method for Soil Particle-Size Determination to Accompany Soil-Quality Analyses". Soil Science Society of America Journal, 65(3), pp. 849-852. 2001.

[9] Wen, B., Aydin, A., Duzgoren-Aydin, N, S. "A comparative study of particle size analyses by sieve-hydrometer and laser diffraction methods". Geotechnical Testing Journal, 25(4), pp. 434-442. 2002.

[10] Emeka, A. E. "Effects of different dispersing solution on the properties of soil particle during hydrometer test". International Journal of Scientific \& Engineering Research, 6(6), pp. 1765-1776. 2015.

https://doi.org/10.4172/2165-784X.1000173

[11] Vitton, S. J., Sadler, L. Y. "Particle-size analysis of soils using laser light scattering and X-ray absorption technology". Geotechnical Testing Journal, 20(1), pp. 63-73. 1997. https://doi.org/10.1520/GTJ11421J

[12] Gee, G. W., Bauder, J. W. "Particle size analysis by hydrometer: a simplified method for routine textural analysis and a sensitivity test of measurement parameters". Soil Science Society of America Journal, 43(5), pp. 1004-1007. 1979.

[13] Bouyoucos, G. J. "The hydrometer as a new method for the mechanical analysis of soils". Soil Science, 23(5), pp. 343-352. 1927. https://doi.org/10.1097/00010694-192705000-00002

[14] Deshpande, V. V., Telang, M. S. "Pipet method of sedimentation analysis. Rapid determination of distribution of particle size". Analytical Chemistry, 22(6), pp. 840-841. 1950. https://doi.org/10.1021/ac60042a033

[15] Konert, M., Vandenberghe, J. "Comparison of laser grain size analysis with pipette and sieve analysis: A solution for the underestimation of the clay fraction". Sedimentology, 44(3), pp. 523-535. 1997. https://doi.org/10.1046/j.1365-3091.1997.d01-38.x

[16] Rodriguez, J. G., Uriarte, A. "Laser diffraction and dry-sieving grain size analyses undertaken on fine- and medium-grained sandy marine sediments: A note". Journal of Coastal Research, 25(1), pp. 257-264. 2009. https://doi.org/10.2112/08-1012.1

[17] Fisher, P., Aumann, C., Chia, K., O'Halloran, N., Chandra, S. "Adequacy of laser diffraction for soil particle size analysis". PLOS ONE, 12(5), 2017. https://doi.org/10.1371/journal.pone.0176510 
[18] Hildebrand, H., Row, G. "Laser light scattering in particle size analysis". The American Ceramic Society Bulletin, 74(7), pp. 49-52. 1995.

[19] Singer, J. K., Anderson, J. B., Ledbetter, M. T., McCave, I. N., Jones, K. P., Wright, R. "An assessment of analytical techniques for the size analysis of fine-grained sediments". Journal of Sedimentary Petrology, 58(3), pp. 534-543. 1988.

[20] Ma, Z., Merkus, H. G., De Smet, J. G. A. E., Heffels, C., Scarlett, B. "New developments in particle characterization by laser diffraction: size and shape". Powder Technology, 111(1-2), pp. 66-78. 2000. https://doi.org/10.1016/S0032-5910(00)00242-4

[21] Jacobsz, S. W., Day, P. "Are we getting what we pay for from geotechnical laboratories?" Journal of South African Institution of Civil Engineering, 16(4), pp. 8-11. 2008.
[22] TMH1:1986. Standard methods of testing road construction materials. Method A6. Pretoria: National Transport Commission. 1986.

[23] SANS, 2014. Civil engineering test methods. SABS Standard Division Designation 3001 Part GR3, SABS, Pretoria. 2014.

[24] British Standard, BS, 1990. "Methods of test for soils for civil engineering purposes". BS Designation 1377 Part 2, BSI, London. 1990.

[25] Indian Standard IS, 1985. Methods of tests for soils. IS Designation 2720 Part IV, BIS, New Delhi.

[26] International Soil Reference and Information Centre, ISRIC, 2002. Procedures for soil analysis. Technical paper 9, The Netherlands. 2002. 